\title{
A Stability Estimate for Robin Boundary Coefficients in Stokes Fluid Flows
}

\author{
Phan Quang Sang and Nguyen Thuy Dung \\ Faculty of Information Technology, Vietnam National University of Agriculture, Hanoi \\ 131000, Vietnam
}

\begin{abstract}
In this report, we examine the unsteady Stokes equations with nonhomogeneous boundary conditions. As an application of a Carleman estimate, we first establish log type stabilities for the solution of the equations from either an interior measurement of the velocity, or a boundary observation depending on the trace of the velocity and of the Cauchy stress tensor measurements on a part of the boundary. We then consider the inverse problem of determining the timeindependent Robin coefficient from a measurement of the solution and of Cauchy data on a sub-boundary.
\end{abstract}

\section{Keywords}

Inverse problems, Carleman inequality, Stokes equation, Stability estimate

AMS Classification: 35R30, 76D07

\section{Introduction}

The Stokes equations are famous equations that describe incompressible fluid flows where the advective inertial forces are small compared with the viscous forces (also called creeping flow). Such a flow is characterized by the property by which the fluid velocities are very slow, while the viscosities are very large, or the length-scales of the flow are very small.

The Stokes equations can be applied to many situations occurring in nature, in technology, and in the modeling of biological problems, for examples, the swimming flow of microorganisms, the flow of lava, the motion of paint, or the flow viscous polymers generally (Dusenbery, 2011), blood flow in the cardiovascular system (Vignon-Clementel et al., 2006), and airflow in the lungs

Received: May 28, 2018 (Baffico et al., 2010).

In this paper we consider the unsteady Stokes equations which can be modeled as following. Let $\Omega$ be a bounded open nonempty subset of $\square^{N}(N=2$ or $N=3)$. 
For some $T>0$, we denote $Q=(0, \mathrm{~T}) \times \Omega$ and consider a velocity-pressure pair $(v, p) \in L^{2}\left(0, \mathrm{~T} ; \mathrm{H}^{2}(\Omega)\right) \cap H^{1}\left(0, \mathrm{~T} ; L^{2}(\Omega)\right) \times L^{2}\left(0, \mathrm{~T} ; \mathrm{H}^{1}(\Omega)\right)$ solution to the following unsteady Stokes equations:

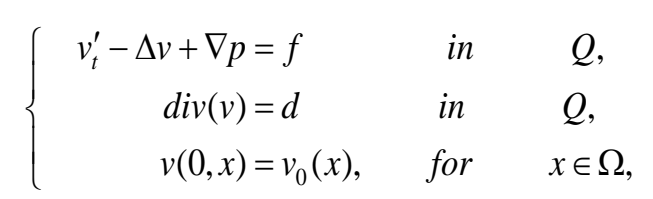

where $f \in \mathrm{L}^{2}(Q)$ is an applied body force and $d \in L^{2}\left(0, \mathrm{~T} ; \mathrm{H}^{1}(\Omega)\right)$.

We notice that the existence of the solution of the Stokes equation (1) is not guaranteed in general. However, it is guaranteed in certain Sobolev spaces under specific conditions; see for example an inf-sup condition (Bramble, 2003; Necas, 2012). In this paper, we will not go into this issue but will focus on the stability of the solution and on an inverse problem of determining a friction boundary coefficient.

Moreover, we need an additional observation to ensure the uniqueness of the solution. There are two main ways of giving such an observation: it is given either by the value of the velocity $v$ in an (arbitrary small) open nonempty subset $\omega \subset \Omega$, or by the Cauchy data $(v, \sigma(v, p) \mathrm{n})$ on a part of the boundary. That is, either

$$
\begin{aligned}
& v(t, x)=v_{o b s}(t, x) \text { in } Q_{\omega}:=(0, \mathrm{~T}) \times \omega, \\
& \text { or }\left\{\begin{array}{rll}
v=g_{D} & \text { on } & (0, \mathrm{~T}) \times \Gamma_{o b s} \\
\sigma(v, p) \mathrm{n}=g_{N} & \text { on } & (0, \mathrm{~T}) \times \Gamma_{o b s}
\end{array}\right.
\end{aligned}
$$

Here, $n$ is the outward unit normal to $\partial \Omega$ which is assumed to be of class $C^{2}$, and the stress tensor is defined by $\sigma(v, p) \stackrel{\text { def }}{=} \eta D(v)-p I$, where $\eta$ is a constant which represents the kinematic viscosity of the fluid we consider, $D(v) \stackrel{\text { def }}{=} \frac{1}{2}\left(\nabla v+{ }^{t} \nabla v\right)$ is the symmetrized gradient, and $I$ is the identity matrix. The uniqueness of the corresponding pair $(v, p)$ is guaranteed by a unique continuation result for the Stokes equations proven in Fabre and Lebeau (1996).

We show in this work the main following results. The first result is an estimate of the solution with respect to the initial data. Then, the second result is the global stability of the solution when we locally change the initial local data. The last result is the stability of a boundary coefficient, called the Robin coefficient, on the unobservable part of the initial data when we change the local data.

The method that we use in this work is based on the construction of an appropriate Carleman estimate for the unsteady Stokes Eq. (1). This method is widely used in many works, including Boulakia et al. (2013) and Badra et al. (2016). However, these works were for steady Stokes equations, or for two dimensions $N=2$. The results of this paper are presented for Stokes equations with time, and in three dimensions $N=3$.

In the following and throughout this work, $C>0$ denotes a generic constant which, unless otherwise stated, only depends on the geometry of and may change from line to line.

Theorem 1.1. Assume that $(v, p) \in L^{2}\left(0, \mathrm{~T} ; \mathrm{H}^{2}(\Omega)\right) \cap H^{1}\left(0, \mathrm{~T} ; L^{2}(\Omega)\right) \times L^{2}\left(0, \mathrm{~T} ; \mathrm{H}^{1}(\Omega)\right)$ is the solution of the Stokes $E q$. (1) such that $\|v\|_{L^{2}\left(0, \mathrm{~T} ; \mathrm{H}^{2}(\Omega)\right)}+\|p\|_{L^{2}\left(0, \mathrm{~T} ; \mathrm{H}^{1}(\Omega)\right)} \leq M$ for some $M>0$. Then there exists a constant $C>0$ such that we have the following estimates: 


$$
\|v\|_{L^{2}(Q)} \leq C \frac{M}{\ln \left(1+\frac{M}{\|f\|_{L^{2}(Q)}+\|d\|_{L^{2}\left(0, T ; \mathrm{H}^{1}(\Omega)\right)}+\|v\|_{\mathrm{L}^{2}\left(Q_{\omega}\right)}}\right)},
$$

and

$$
\|v\|_{L^{2}(Q)} \leq C \frac{M}{\ln \left(1+\frac{M}{\|f\|_{L^{2}(Q)}+\|d\|_{L^{2}(Q)}+\|v\|_{L^{2}\left(0, T ; H^{3 / 2}\left(\Gamma_{o b s}\right)\right)}+\|\sigma(v, p) \mathrm{n}\|_{L^{2}\left(0, T ; H^{1 / 2}\left(\Gamma_{o b s}\right)\right)}}\right)} .
$$

Moreover,

$$
\begin{aligned}
& \|\operatorname{curl}(v)\|_{\left(\mathrm{L}^{2}(Q)\right)^{2 N-3}}+\|p-\operatorname{div}(v)\|_{L^{2}(Q)} \\
& \leq C \frac{M}{\left[\ln \left(1+\frac{M}{\|f\|_{L^{2}(Q)}+\|d\|_{L^{2}(Q)}+\|v\|_{L^{2}\left(0, T ; H^{3 / 2}\left(\Gamma_{o b s}\right)\right)}+\|\sigma(v, p) \mathrm{n}\|_{L^{2}\left(0, T ; H^{1 / 2}\left(\Gamma_{o b s}\right)\right)}}\right)\right]^{\frac{1}{2}} .}
\end{aligned}
$$

We notice that the (4), (5), and (6) estimates will be further proven by Theorem 3.1.2 and Theorem 3.2.3.

As an application of the above theorem, we can obtain the stability estimate for the Stokes equations (1).

Assume that $\left(v_{i}, p_{i}\right) \in L^{2}\left(0, \mathrm{~T} ; \mathrm{H}^{2}(\Omega)\right) \cap H^{1}\left(0, \mathrm{~T} ; L^{2}(\Omega)\right) \times L^{2}\left(0, \mathrm{~T} ; \mathrm{H}^{1}(\Omega)\right), i=1,2$, resulting in two solutions for Eq. (1) associated to one of two types of observations:

either

$$
\begin{aligned}
& v_{i}(t, x)=v_{\text {obs }}^{i}(t, x), i=1,2, \quad \text { in } \quad(0, \mathrm{~T}) \times \omega, \\
& \text { or }\left\{\begin{array}{rlll}
v_{i}=g^{i}{ }_{D}, i=1,2, & \text { on } & & (0, \mathrm{~T}) \times \Gamma_{o b s} . \\
\sigma\left(v_{i}, p_{i}\right) \mathrm{n}=g_{N}^{i}, i=1,2, & \text { on } & & (0, \mathrm{~T}) \times \Gamma_{o b s}
\end{array}\right. \text {. }
\end{aligned}
$$

Then we have the following result (proven with equations 43, 44, and 45):

Theorem 1.2. Assume that $\left(v_{i}, p_{i}\right) \in L^{2}\left(0, \mathrm{~T} ; \mathrm{H}^{2}(\Omega)\right) \cap H^{1}\left(0, \mathrm{~T} ; L^{2}(\Omega)\right) \times L^{2}\left(0, \mathrm{~T} ; \mathrm{H}^{1}(\Omega)\right), i=1,2$,

There are two solutions for Eq. (1) associated with one of two additional observations given by (7) or (8). Moreover, we suppose that $\left\|v_{1}-v_{2}\right\|_{L^{2}\left(0, \mathrm{~T} ; \mathrm{H}^{2}(\Omega)\right)}+\left\|p_{1}-p_{2}\right\|_{L^{2}\left(0, \mathrm{~T} ; \mathrm{H}^{1}(\Omega)\right)} \leq M$ for some $M>0$. Then there exists a constant $C>0$ such that we have the following estimates:

$$
\left\|v_{1}-v_{2}\right\|_{L^{2}(Q)} \leq C \frac{M}{\ln \left(1+\frac{M}{\left\|v_{1}-v_{2}\right\|_{L^{2}\left(Q_{\omega}\right)}}\right)},
$$

and

$$
\left\|v_{1}-v_{2}\right\|_{L^{2}(Q)} \leq C \frac{M}{\ln \left(1+\frac{M}{\left\|v_{1}-v_{2}\right\|_{L^{2}\left(0, T ; H^{3 / 2}\left(\Gamma_{o b s}\right)\right)}+\left\|\sigma\left(v_{1}, p_{1}\right) \mathrm{n}-\sigma\left(v_{2}, p_{2}\right) \mathrm{n}\right\|_{L^{2}\left(0, T ; H^{1 / 2}\left(\Gamma_{o b s}\right)\right)}}\right)} .
$$


Moreover, we have

$$
\begin{aligned}
\left\|\operatorname{curl}\left(v_{1}-v_{2}\right)\right\|_{\left(\mathrm{L}^{2}(Q)\right)^{2 N-3}}+\left\|p_{1}-p_{2}\right\|_{\mathrm{L}^{2}(Q)} \\
\leq C \frac{M}{\left[\ln \left(1+\frac{M}{\left\|v_{1}-v_{2}\right\|_{\mathrm{L}^{2}\left(0, T ; H^{3 / 2}\left(\Gamma_{o b s}\right)\right)}+\left\|\sigma\left(v_{1}, p_{1}\right) \mathrm{n}-\sigma\left(v_{2}, p_{2}\right) \mathrm{n}\right\|_{\mathrm{L}^{2}\left(0, T ; H^{1 / 2}\left(\Gamma_{o b s}\right)\right)}}\right)\right]^{\frac{1}{2}}}
\end{aligned}
$$

We notice that the results of this theorem lead to the uniqueness of the solution $(v, p)$ of Eq. (1): if $v_{1}=v_{2}$ in $Q_{\omega}$ then $v_{1}=v_{2}$ in $Q$, or if the Cauchy data $\left(v_{1}, \sigma\left(v_{1}, p_{1}\right) \mathrm{n}\right)=\left(v_{2}, \sigma\left(v_{2}, p_{2}\right) \mathrm{n}\right)$ on $(0, \mathrm{~T}) \times \Gamma_{o b s}$, then $v_{1}=v_{2}$ in $Q$. This matches the unique continuation result given in Fabre and Lebeau (1996).

Similar stability estimates were given for the Navier-Stokes equations, as in the paper by Badra et al. (2016).

An important purpose of this article is to prove the stability in the determination of the Robin boundary coefficient from the value of velocity $v$ and the Cauchy data $(v, \sigma(v, p) n)$ on a part of the boundary. This kind of inverse problem is very significant in general in corrosion detection: the determination of the Robin coefficient on the inaccessible portion of the boundary thanks to electrostatic measurements performed on the accessible boundary part.

We assume that $\Gamma_{0}$ is another open nonempty subset of boundary $\partial \Omega$ such that $\Gamma_{0} \cap \Gamma_{o b s}=\varnothing$.

We suppose that on $\Gamma_{0}$, corresponding to the previous pairs $\left(v_{1}, p_{1}\right),\left(v_{2}, p_{2}\right)$, the fluid has two friction boundary coefficients given by the conditions

$$
\sigma\left(v_{i}, p_{i}\right) \mathrm{n}+\alpha_{i} v_{i}=0, i=1,2 .
$$

The coefficients $\alpha_{i}$ in (12) are called the Robin coefficients. We have the following stability estimate for the Robin coefficients (proven with equations 46, 47, 48, 49, and 50):

Theorem 1.3. Assume that $\left(v_{i}, p_{i}\right) \in L^{2}\left(0, \mathrm{~T} ; \mathrm{H}^{2}(\Omega)\right) \cap H^{1}\left(0, \mathrm{~T} ; L^{2}(\Omega)\right) \times L^{2}\left(0, \mathrm{~T} ; \mathrm{H}^{1}(\Omega)\right), i=1,2$, are two solutions of Eq. (1) associated with the additional observation given by (8). Let $\alpha_{i}, i=1,2$ be the two Robin coefficients given by (12). Let $\mathrm{N}$ be the set $\mathrm{N}=\left\{x \in \Gamma_{0}, v_{1}(x)=v_{2}(x)=0\right\}$ and we assume that $\mathrm{K}$ is a compact of $\Gamma_{0} \backslash \mathrm{N}$ with a nonempty interior, and then let $m>0$ be a constant such that $\max \left(\left|v_{1}\right|,\left|v_{2}\right|\right) \geq m$ on $\mathrm{K}$.

Moreover, we suppose that $\left\|v_{1}-v_{2}\right\|_{L^{2}\left(0, \mathrm{~T} ; \mathrm{H}^{2}(\Omega)\right)}+\left\|p_{1}-p_{2}\right\|_{L^{2}\left(0, \mathrm{~T} ; \mathrm{H}^{1}(\Omega)\right)} \leq M$ for some $M>0$.

Then there exists a constant $C>0$ such that we have the following estimates

$$
\begin{aligned}
& \left\|\alpha_{1}-\alpha_{2}\right\|_{\mathrm{L}^{2}((0, T) \times \mathrm{K})} \\
& \leq \frac{C}{m} \frac{M}{\left[\ln \left(1+\frac{M}{\left\|v_{1}-v_{2}\right\|_{L^{2}\left(0, T ; H^{3 / 2}\left(\Gamma_{o b s}\right)\right)}+\left\|\sigma\left(v_{1}, p_{1}\right) \mathrm{n}-\sigma\left(v_{2}, p_{2}\right) \mathrm{n}\right\|_{L^{2}\left(0, T ; H^{1 / 2}\left(\Gamma_{o b s}\right)\right)}}\right)\right]^{\frac{1}{4}}}
\end{aligned}
$$

There is a wide collection of mathematical works dealing with inverse boundary coefficient problems. Most of them prove a logarithmic stability estimate for boundary coefficients in stationary Stokes equations (Chaabane et al., 2004; Sincich, 2007; Bellassoued et al., 2008; Cheng et al., 2008) 
or in two dimensions (Boulakia et al., 2013). The paper by Badra et al. (2016) presented the inverse problem of the Robin coefficient for stationary Navier-Stokes equations. The paper by Boulakia et al. (2013) gave stability estimates for the Robin coefficient but in the two dimensional Stokes equations.

Otherwise, the present inverse problem is for unsteady Stokes equations in two or three dimensions. It improves upon several of the previously cited works and so it is new.

Notations Through this paper, $\Omega$ is a nonempty bounded open subset of $\square^{N}$ for $N=2$ or $N=3$, with a boundary $\partial \Omega$ of class $C^{2}$ and $\omega$ is a nonempty open subset of $\Omega$.

For some $T>0$, we denote $Q=(0, \mathrm{~T}) \mathrm{x} \Omega$ and $Q_{\omega}=(0, \mathrm{~T}) \times \omega$.

Let $v$ be a vector field, $v=\left(v_{1}, v_{2}, \ldots, v_{N}\right)$, then we define:

- the gradient of $v$ is $\nabla v=\left(\partial_{x_{j}} v_{i}\right)_{1 \leq i, j \leq N}$,

- the Laplacian of $v$ is $\Delta v=\left(\Delta v_{i}\right)_{i=\overline{1, N}}=\left(\sum_{j=1}^{N} \partial_{x_{j}^{2}}^{2} v_{i}\right)_{i=1, N}$,

- the divergence of $v$ is $\operatorname{div} v=\sum_{i=1}^{N} \partial_{x_{i}} v_{i}$, and

- the curl of $v$ is the vector function is:

$$
\operatorname{curl}(v)=\partial_{x_{1}} v_{2}-\partial_{x_{2}} v_{1} \text { if } N=2 \text {, or } \operatorname{curl}(v)=\left(\begin{array}{l}
\partial_{x_{2}} v_{3}-\partial_{x_{3}} v_{2} \\
\partial_{x_{3}} v_{1}-\partial_{x_{1}} v_{3} \\
\partial_{x_{1}} v_{2}-\partial_{x_{2}} v_{1}
\end{array}\right) \text { if } N=3 \text {. }
$$

\section{Carleman Estimate for Unstaedy Stokes Equations}

The main aim of this section is to prove a Carleman inequality for the non-homogeneous Stokes equations. For that, we first prove a Carleman inequality for a velocity-pressure pair in $\mathrm{L}^{2}\left(0, T ; \mathrm{H}_{0}^{2}(\Omega)\right) \cap \mathrm{H}_{0}^{1}\left(0, \mathrm{~T} ; \mathrm{L}^{2}(\Omega)\right) \times \mathrm{L}^{2}\left(0, T ; \mathrm{H}_{0}^{1}(\Omega)\right)$ and then we use a domain extension argument to recover the non-homogeneous case.

For $T>0$, we recall that $Q=(0, T) \times \Omega$ and $Q_{\omega}=(0, T) \mathrm{x} \omega$ for an open nonempty subset $\omega \subset \Omega$. Let $\psi: \Omega \rightarrow \square$ be a function satisfying

$$
\begin{aligned}
& \psi \in C^{2}(\Omega ; \square), \psi>c_{0} \text { and }|\nabla \psi|>0 \text { in } \Omega \backslash \bar{\omega}, \\
& \psi=c_{0} \text { on } \partial \Omega
\end{aligned}
$$

for some positive constant $c_{0}>0$. For the existence of such a function, see Tucsnak and Weiss (2009), for instance.

Then we introduce the weight functions:

$$
\begin{aligned}
& \varphi(t, x)=\frac{e^{\lambda \psi(x)}}{t(T-t)}, \hat{\varphi}(t)=\frac{1}{t(T-t)}, \alpha(t, x)=\frac{e^{\lambda \psi(x)}-e^{2 \lambda\|\psi\|_{C(\bar{\Omega})}}}{t(T-t)}, \\
& \varphi_{0}=\varphi_{0}(t)=\hat{\varphi}(t) e^{\lambda c_{0}}, \alpha_{0}=\alpha_{0}(t)=\hat{\varphi}(t) e^{\lambda c_{0}}-e^{2 \lambda\|\psi\|_{C(\bar{\Omega})}}
\end{aligned}
$$




\section{Carleman estimate in the case of homogeneous boundary data}

Due to a result from Badra et al. (2016), we can easily get the following result.

Theorem 2.1.1. Let $k \in\{0,1\}, F \in \mathrm{L}^{2}(\mathrm{Q})$, and $G \in \mathrm{L}^{2}(Q)$, then there exists $C>0, \lambda>1$, and $\hat{s}>1$ such that for all $\lambda \geq \lambda$ and $s \geq \hat{s}$, the solution $v \in \mathrm{L}^{2}(Q)$ of

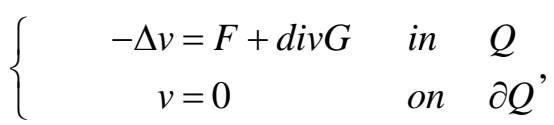

satisfies the following inequality: $\int_{Q}\left(\varphi^{k-1}|\nabla v|^{2}+s^{2} \varphi^{k+1}|v|^{2}\right) e^{2 s \alpha} \mathrm{d} x \mathrm{~d} t$

$$
\leq C\left(\left\|s^{-1 / 2} \varphi^{k / 2-1} F e^{s \alpha}\right\|_{L^{2}(Q)}^{2}+\left\|s^{1 / 2} \varphi^{k / 2} G e^{s \alpha}\right\|_{L^{2}(Q)}^{2}+\int_{Q_{\omega}} s^{2} \varphi^{k+1} v^{2} e^{2 s \alpha} d x d t\right) .
$$

We recall here a Carleman estimate for homogeneous Stokes equations cited from Imanuvilov and Yamamoto (2003).

Theorem 2.1.2. Let $F \in \mathrm{L}^{2}(\mathrm{Q})$ and $G \in \mathrm{L}^{2}(Q)$, then there exists $C>0, \lambda>1$ and $\hat{s}>1$ such that for all $\lambda \geq \lambda$ and $s \geq \hat{s}$, the solution $v \in \mathrm{L}^{2}(Q)$ of

$$
\begin{aligned}
& \left\{\begin{array}{cl}
v_{t}^{\prime}-\Delta v=F+\operatorname{div} G & \text { in } Q \\
v=0 & \text { on } \partial Q
\end{array},\right. \text { satisfies the following inequality: } \\
& \int_{Q}\left(s^{-1} \varphi^{-1}|\nabla v|^{2}+s \varphi|v|^{2}\right) e^{2 s \alpha} \mathrm{d} x \mathrm{~d} t \leq C\left(\left\|F e^{s \alpha}\right\|_{L^{2}(Q)}^{2}+\left\|G e^{s \alpha}\right\|_{L^{2}(Q)}^{2}+\int_{Q_{\omega}} s \varphi v^{2} e^{2 s \alpha} d x d t\right),
\end{aligned}
$$

where the constant $C>0$ is dependent continuously on $\lambda, \mathrm{k}$ and is independent of $s$.

Using the two previous theorems, we can get a Carleman estimate for the unsteady Stokes equations with homogenous boundary data.

Theorem 2.1.3. There exists $C>0, \lambda>1$ and $\hat{s}>1$ such that for all $\lambda>\lambda$ and $s>\hat{s}$, and for all $(v, p) \in \mathrm{L}^{2}\left(0, T ; \mathrm{H}_{0}^{2}(\Omega)\right) \cap \mathrm{H}_{0}^{1}\left(0, T ; \mathrm{L}^{2}(\Omega)\right) \times \mathrm{L}^{2}\left(0, T ; \mathrm{H}_{0}^{1}(\Omega)\right)$, the following inequalities hold:

$$
\begin{aligned}
& \int_{Q}\left(|\nabla v|^{2}+s \varphi|\operatorname{curl} v|^{2}+s^{2} \varphi^{2}|v|^{2}\right) e^{2 s \alpha} \mathrm{d} x \mathrm{~d} t \\
& \quad \leq C\left(\int_{Q_{\omega}} s^{3} \varphi^{3}|v|^{2} e^{2 s \alpha} \mathrm{d} x \mathrm{~d} t+\left\|\left(v_{t}^{\prime}-\Delta v+\nabla p\right) e^{s \alpha}\right\|_{L^{2}(\mathrm{Q})}^{2}+\left\|(\nabla \operatorname{div} v) s^{-1 / 2} \varphi^{-1 / 2} e^{s \alpha}\right\|_{\mathrm{L}^{2}(\mathrm{Q})}^{2}\right), \\
& \text { and } \int_{Q}\left(s \varphi|\operatorname{div} v-p|^{2} e^{2 s \varphi}\right) d x d t \leq C\left(\int_{Q}|\nabla p-\Delta v|^{2} e^{2 s \varphi} d x d t+\int_{Q_{\omega}} s \varphi|\operatorname{div} v-p|^{2} e^{2 s \varphi} d x d t\right)
\end{aligned}
$$

Proof: We set $f \stackrel{\text { def }}{=} v_{t}^{\prime}-\Delta v+\nabla p$. Easy calculations yield:

$$
\begin{aligned}
& (\operatorname{curl} v)_{t}^{\prime}-\Delta(\operatorname{curl} v)=\operatorname{curl} f \quad \text { in } \quad Q, \\
& -\Delta v=\operatorname{curl}(\operatorname{curl} v)-\nabla(\operatorname{div} v) \quad \text { in } \quad Q, \\
& -\Delta(\operatorname{div} v-p)=\operatorname{div} f
\end{aligned}
$$


To get (18), we just apply Theorem 2.1.1 for $k=0$ to Eq. (21).

Now we introduce a relatively compact open subset $\omega_{0}$ of $\omega$ and apply Theorem 2.1.2 (the inequality (16)) for $k=0$ to Eq. (19) to obtain:

$$
\begin{aligned}
& \int_{Q} s^{-1} \varphi^{-1}|\nabla(\operatorname{curl} v)|^{2} \mathrm{e}^{2 s \alpha} \mathrm{d} x \mathrm{~d} t+\int_{Q} s \varphi|\operatorname{curl} v|^{2} \mathrm{e}^{2 s \alpha} \mathrm{d} x \mathrm{~d} t \\
& \leq C\left(\int_{Q_{\infty}} s \varphi|\operatorname{curl} v|^{2} e^{2 s \alpha} \mathrm{d} x \mathrm{~d} t+\left\|\left(v_{t}^{\prime}-\Delta v+\nabla p\right) e^{s \alpha}\right\|_{L^{2}(\mathrm{Q})}^{2}\right), \\
& \leq C\left(\int_{Q}\left(s^{-1} \lambda^{-2} e^{-\lambda \psi}|\nabla d i v v|^{2}+\lambda^{-2}|\nabla p-\Delta v|^{2}\right) e^{2 s e^{\lambda \psi}} d x d t+\int_{Q_{\omega}} s^{3} \lambda^{2} e^{3 \lambda \psi} d x d t\right) .
\end{aligned}
$$

In the last inequality, let us estimate the local term in curl $v$ by a local term in $v$. For that, we introduce the function $\rho \in C_{0}^{\infty}(\omega)$ such that $0 \leq \rho \leq 1$ and $\rho=1$ in $\omega_{0}$. Using an integration by parts in $Q_{\omega}$, we get

$$
\begin{aligned}
\int_{Q_{0}} s \varphi|\operatorname{curl} v|^{2} e^{2 s \alpha} \mathrm{d} x \mathrm{~d} t & \leq s \int_{Q_{\omega}} \rho \varphi|\operatorname{curl} v|^{2} e^{2 s \alpha} \mathrm{d} x \mathrm{~d} t=s \int_{Q_{\omega}} \operatorname{curl}\left(\rho \varphi e^{2 s \alpha} \operatorname{curlv}\right) \mathrm{d} x \mathrm{~d} t \\
& \leq C\left(\int_{Q_{\omega}} s^{2} \varphi^{2} e^{2 s \alpha}|v||\operatorname{curl} v| \mathrm{d} x \mathrm{~d} t+\int_{Q_{\omega}} s \varphi e^{2 s \alpha}|v||\nabla(\operatorname{curl} v)| \mathrm{d} x \mathrm{~d} t\right)
\end{aligned}
$$

and then with the Cauchy- Schwarz inequality:

$$
\begin{aligned}
\int_{Q_{\infty}} s \varphi|\operatorname{curl} v|^{2} e^{2 s e^{\lambda \mu} \mathrm{d} x \mathrm{~d} t} & \leq \varepsilon \int_{Q_{\omega}}\left(s^{-1} \varphi^{-1} e^{2 s \alpha}|\nabla(\operatorname{curl} v)|^{2}+s \varphi e^{2 s \alpha}|\operatorname{curl} v|^{2}\right) \mathrm{d} x \mathrm{~d} t \\
& +\frac{C}{\varepsilon} s^{3} \int_{Q_{\omega}} \varphi^{3} e^{2 s \alpha}|\nu|^{2} \mathrm{~d} x \mathrm{~d} t
\end{aligned}
$$

By combining (22) with the above inequality for small enough values of $\varepsilon>0$, we obtain

$$
\begin{aligned}
& \int_{Q} s^{-1} \varphi^{-1} \mid \nabla\left.(\operatorname{curl} v)\right|^{2} \mathrm{e}^{2 s \alpha} \mathrm{d} x \mathrm{~d} t+\int_{Q} s \varphi|\operatorname{curl} v|^{2} \mathrm{e}^{2 s \alpha} \mathrm{d} x \mathrm{~d} t \\
& \leq C\left(\int_{Q_{\omega}} s^{3} \varphi^{3}|v|^{2} e^{2 s \alpha} \mathrm{d} x \mathrm{~d} t+\left\|\left(v_{t}^{\prime}-\Delta v+\nabla p\right) e^{s \alpha}\right\|_{L^{2}(Q)}^{2}\right)
\end{aligned}
$$

Finally, (17) is obtained by first applying (16) (for $k=1$ ) to Eq. (20) and then using the estimate of curlv given by (24).

\section{Carleman estimate in the case of non-homogeneous boundary data}

In this section, we prove a Carleman inequality for the Stokes equations with non-homogenous boundary data. We consider the equation:

$$
\left\{\begin{aligned}
v_{t}^{\prime}-\Delta v+\nabla p=f & \text { in } \quad Q, \\
\operatorname{div} v=\mathrm{d} & \text { in } \quad Q .
\end{aligned}\right.
$$

We recall that $C>0$ denotes a generic constant depending only on the geometry of the boundary and is independent of $s$. 
Theory 2.2.1. There exists $C>0, \lambda>1$ and $\hat{s}>1$ such that for all $\lambda>\lambda$ and $s \geq \hat{s}$, every solution $(v, p) \in \mathrm{L}^{2}\left(0, T ; \mathrm{H}^{2}(\Omega)\right) \cap \mathrm{H}^{1}\left(0, T ; \mathrm{L}^{2}(\Omega)\right) \times \mathrm{L}^{2}\left(0, T ; \mathrm{H}^{1}(\Omega)\right)$ of $(25)$ satisfies:

$$
\begin{aligned}
& \int_{Q}\left(|\nabla v|^{2}+s \varphi|\operatorname{curl} v|^{2}+s^{2} \varphi^{2}|v|^{2}\right) e^{2 s \alpha} \mathrm{d} x \mathrm{~d} t \\
& \leq C\left(\int_{Q_{\omega}} s^{3} \varphi^{3}|v|^{2} e^{2 s \alpha} \mathrm{d} x \mathrm{~d} t+\left\|\mathrm{e}^{s \alpha} f\right\|_{\mathrm{L}^{2}(\mathrm{Q})}^{2}+\left\|(\nabla d) s^{-1 / 2} \varphi^{-1 / 2} e^{s \alpha}\right\|_{\mathrm{L}^{2}(\mathrm{Q})}^{2}\right) \\
& \quad+C e^{2 s \alpha_{0}}\left(\|v\|_{\mathrm{L}^{2}\left(0, \mathrm{~T} ; \mathrm{H}^{2}(\Omega)\right.}^{2}+\|p\|_{\mathrm{L}^{2}\left(0, \mathrm{~T} ; \mathrm{H}^{1}(\Omega)\right.}^{2}\right)
\end{aligned}
$$

and

$$
\begin{aligned}
& \int_{Q}\left(s \varphi|d-p|^{2} e^{2 s \varphi}\right) d x d t \leq C\left(\int_{Q}|f|^{2} e^{2 s \varphi} d x d t+\int_{Q_{\omega}} s \varphi|d-p|^{2} e^{2 s \varphi} d x d t\right) \\
& +C e^{2 s \alpha_{0}}\left(\|v\|_{\mathrm{L}^{2}\left(0, \mathrm{~T} ; \mathrm{H}^{2}(\Omega)\right.}^{2}+\|p\|_{\mathrm{L}^{2}\left(\mathrm{O}, \mathrm{T} ; \mathrm{H}^{1}(\Omega)\right.}^{2}\right) .
\end{aligned}
$$

Proof: Let $\Omega$ be a bounded domain of $\square^{N}\left(N=2\right.$ or $N=3$ ) of class $C^{2}$ such that $\Omega$ is relatively compact in $\Omega$. We denote $Q=(0, T) \times \Omega$. We extend $\psi$ to $\Omega$ (while keeping the same name) in such a way that:

$$
\begin{aligned}
& \psi \in C^{2}(\Omega ; \square), \quad \psi>0 \text { and }|\nabla \psi|>0 \quad \text { in } \Omega \backslash \bar{\Omega}, \\
& \psi \equiv c_{0} \quad \text { on } \partial \Omega, \quad 0<\psi<c_{0} \text { in } \Omega \backslash \bar{\Omega}, c_{0}<\psi \text { in } \Omega, \\
& \text { and we denote } \alpha_{0}=\alpha_{0}(t)=\hat{\varphi}(t) e^{\lambda c_{0}}-e^{2 \lambda\|\psi\|_{c(\bar{\Omega})}} \text {. }
\end{aligned}
$$

Let $E$ be a linear continuous map from $\mathrm{L}^{2}\left(0, T ; \mathrm{H}^{2}(\Omega)\right) \cap \mathrm{H}^{1}\left(0, T ; \mathrm{L}^{2}(\Omega)\right) \times \mathrm{L}^{2}\left(0, T ; \mathrm{H}^{1}(\Omega)\right)$ into $\mathrm{L}^{2}\left(0, T ; \mathrm{H}_{0}^{2}(\tilde{\Omega})\right) \cap \mathrm{H}_{0}^{1}\left(0, T ; \mathrm{L}^{2}(\Omega)\right) \times \mathrm{L}^{2}\left(0, T ; \mathrm{H}_{0}^{1}(\tilde{\Omega})\right)$ such that $E(v, p) \equiv(v, p)$ in $Q$ (given by Stein's Theorem, see Adams (2003)), and we define $(\tilde{v}, \tilde{p}) \stackrel{\operatorname{def}}{=} E(v, p)$. Then the pair $(\tilde{v}, \tilde{p})=E(v, p)$ is the solution to the system:

$$
\left\{\begin{array}{rlrl}
\tilde{v}_{t}^{\prime}-\Delta \tilde{v}+\nabla \tilde{p}=\tilde{f} & & \text { in } & \tilde{Q}, \\
\operatorname{div} \tilde{v}=\tilde{d} & \text { in } & \tilde{Q}, \\
\tilde{v}=0 & \text { in } & \partial \tilde{Q}, \\
\frac{\partial \tilde{v}}{\partial n}=0 & \text { in } & \partial \tilde{Q}, \\
\tilde{p}=0 & \text { in } & \partial \tilde{Q},
\end{array}\right.
$$

where $\tilde{f} \in \mathrm{L}^{2}(\tilde{Q})$ and $\tilde{d} \in \mathrm{L}^{2}\left(0, T ; \mathrm{H}^{1}(\tilde{\Omega})\right)$ are given by $\tilde{f}=f$ and $\tilde{d}=d$ in $Q$, and by $\tilde{f}=\tilde{v}_{t}^{\prime}-\Delta \tilde{v}+\nabla \tilde{p}$ and $\tilde{d}=\operatorname{div} \tilde{v}$ in $\tilde{Q} \backslash \bar{Q}$. From the continuity of the extension operator $E$, we have:

$$
\|\tilde{f}\|_{\mathrm{L}^{2}(\tilde{Q} \backslash Q)}^{2}+\|\tilde{d}\|_{\mathrm{L}^{2}\left(0, T ; \mathrm{H}^{1}(\tilde{\Omega} \backslash \bar{\Omega})\right)}^{2} \leq C\left(\|v\|_{\mathrm{L}^{2}\left(0, \mathrm{~T} ; \mathrm{H}^{2}(\Omega)\right.}^{2}+\|p\|_{\mathrm{L}^{2}\left(0, \mathrm{~T} ; \mathrm{H}^{1}(\Omega)\right.}^{2}\right) \text {. }
$$

Next, by applying estimate (17) of Theorem 2.1.3, we have: 


$$
\begin{aligned}
& \int_{\tilde{Q}}\left(|\nabla \tilde{v}|^{2}+s \varphi|\operatorname{cur} \mathrm{v}|^{2}+s^{2} \varphi^{2}|\tilde{v}|^{2}\right) e^{2 s \alpha} \mathrm{d} x \mathrm{~d} t \\
& \leq C\left(\int_{Q_{\omega}} s^{3} \varphi^{3}|\tilde{v}|^{2} e^{2 s \alpha} \mathrm{d} x \mathrm{~d} t+\left\|e^{s \alpha} \tilde{f}\right\|_{L^{2}(\tilde{Q})}^{2}+\left\|(\nabla \tilde{d}) s^{-1 / 2} \varphi^{-1 / 2} e^{s \alpha}\right\|_{\mathrm{L}^{2}(\tilde{Q})}^{2}\right)
\end{aligned}
$$

Moreover, for $s \geq \hat{s}>1$, applying the estimates (28) and (29), we have:

$$
\begin{aligned}
& \left\|e^{s \alpha} \tilde{f}\right\|_{\mathrm{L}^{2}(\tilde{Q} \backslash Q)}^{2}+\left\|(\nabla \tilde{d}) s^{-1 / 2} \varphi^{-1 / 2} e^{s \alpha}\right\|_{\mathrm{L}^{2}(\tilde{Q} \backslash Q)}^{2} \\
& \leq e^{2 s \alpha}\left(\|\tilde{f}\|_{\mathrm{L}^{2}(\tilde{Q} \backslash Q)}^{2}+\|\tilde{d}\|_{\mathrm{L}^{2}\left(0, T ; \mathrm{H}^{1}(\tilde{\Omega} \backslash \Omega)\right)}^{2}\right) \leq C e^{2 s \alpha_{0}}\left(\|v\|_{\mathrm{L}^{2}\left(0, \mathrm{~T} ; \mathrm{H}^{2}(\Omega)\right.}^{2}+\|p\|_{\mathrm{L}^{2}\left(0, \mathrm{~T} ; \mathrm{H}^{1}(\Omega)\right.}^{2}\right) .
\end{aligned}
$$

Using (30) and (31), we have the proof for (26).

To prove (27), we apply (18) of Theorem 2.1.3 to $(\tilde{v}, \tilde{p})$ to get:

$$
\begin{aligned}
& \int_{Q}\left(s \varphi|\operatorname{div} \tilde{v}-\tilde{p}|^{2} e^{2 s \varphi}\right) d x d t \leq C\left(\int_{\tilde{Q}}|\tilde{f}|^{2} e^{2 s \varphi} d x d t+\int_{Q_{\omega}} s \varphi|\operatorname{div} \tilde{v}-\tilde{p}|^{2} e^{2 s \varphi} d x d t\right) \\
& \leq C\left(\int_{Q}|f|^{2} e^{2 s \varphi} d x d t+\int_{Q_{\omega}} s \varphi|\operatorname{div} v-p|^{2} e^{2 s \varphi} d x d t\right)+C e^{2 s \alpha_{0}}\left(\|v\|_{L^{2}\left(0, \mathrm{~T} ; \mathrm{H}^{2}(\Omega)\right.}^{2}+\|p\|_{\mathrm{L}^{2}\left(0, \mathrm{~T} ; \mathrm{H}^{1}(\Omega)\right.}^{2}\right) .
\end{aligned}
$$

\section{Stability Estimates for Unsteady Stokes Equations and The Inverse Problem of the Robin Coefficient}

In this section, we show stability estimates for unsteady Stokes equations corresponding to a distributed observation or a boundary observation, which allow proving the main results announced in Theorem 1.1 and Theorem 1.2. Then, we can apply them to the inverse problem of determining the Robin boundary coefficient presented in Theorem 1.3.

\section{Estimates for the solutions with a distributed observation}

In this subsection, we use the Carleman inequalities given in Theorem 2.4 to obtain several stability estimates with a distributed observation.

Theorem 3.1.1. There exists $\hat{\lambda}>1$ and $\hat{s}>1$ such that all $\lambda>\hat{\lambda}$ and all $s \geq \hat{s}$, with large enough $c^{*}$, result in every solution $(v, p) \in \mathrm{L}^{2}\left(0, T ; \mathrm{H}^{2}(\Omega)\right) \cap \mathrm{H}^{1}\left(0, T ; \mathrm{L}^{2}(\Omega)\right) \times \mathrm{L}^{2}\left(0, T ; \mathrm{H}^{1}(\Omega)\right)$ of Eq. (25) satisfying:

$$
\|v\|_{\mathrm{L}^{2}(\mathrm{Q})} \leq e^{s e^{e^{*} \lambda}}\left(\|f\|_{\mathrm{L}^{2}(Q)}+\|d\|_{\mathrm{L}^{2}\left(0, T ; \mathrm{H}^{1}(\Omega)\right)}+\|v\|_{\mathrm{L}^{2}\left(\mathrm{Q}_{\omega}\right)}\right)+\frac{1}{S}\left(\|v\|_{\mathrm{L}^{2}\left(0, \mathrm{~T} ; \mathrm{H}^{2}(\Omega)\right.}+\|p\|_{\mathrm{L}^{2}\left(0, \mathrm{~T} ; \mathrm{H}^{1}(\Omega)\right.}\right) .
$$

Proof: Let $\psi$ be the function defined by (14). We define the following:

$$
\begin{aligned}
\psi_{\min } & =\min _{(\mathrm{t}, x) \in Q} \psi(\mathrm{t}, x)=\mathrm{c}_{0}, \text { and } \psi_{\max }=\max _{(\mathrm{t}, x) \in Q} \psi(\mathrm{t}, x), \\
\varphi_{1} & =\varphi_{1}(t)=\frac{e^{\lambda \psi \max }}{t(T-t)}, \alpha_{1}=\alpha_{1}(t)=\frac{e^{\lambda \psi \max }-e^{2 \lambda\|\psi\|_{C(\bar{\Omega})}}}{t(T-t)}, \\
\varphi_{0} & =\varphi_{0}(t)=\hat{\varphi}(t) e^{\lambda c_{0}}, \alpha_{0}=\alpha_{0}(t)=\hat{\varphi}(t) e^{\lambda c_{0}}-e^{2 \lambda\|\psi\|_{C(\bar{\Omega})}} .
\end{aligned}
$$


We apply (26) to $(v, p)$ to get

$$
\begin{aligned}
\int_{Q}\left(s \varphi|\operatorname{curl} v|^{2}\right. & \left.+s^{2} \varphi^{2}|v|^{2}\right) e^{2 s \alpha} \mathrm{d} x \mathrm{~d} t \\
& \leq C\left(\int_{Q_{\omega}} s^{3} \varphi_{1}^{3}|v|^{2} e^{2 s \alpha_{1}} \mathrm{~d} x \mathrm{~d} t+\left\|\mathrm{e}^{s \alpha_{1}} f\right\|_{\mathrm{L}^{2}(Q)}^{2}+\left\|(\nabla d) s^{-1 / 2} \varphi_{1}^{-1 / 2} e^{s \alpha_{1}}\right\|_{\mathrm{L}^{2}(Q)}^{2}\right) \\
& +C e^{2 s \alpha_{0}}\left(\|v\|_{\mathrm{L}^{2}\left(0, \mathrm{~T} ; \mathrm{H}^{2}(\Omega)\right.}^{2}+\|p\|_{\mathrm{L}^{2}\left(0, \mathrm{~T} ; \mathrm{H}^{1}(\Omega)\right.}^{2}\right),
\end{aligned}
$$

and then

$$
\begin{aligned}
\int_{Q}\left(s \varphi_{0}|\operatorname{curl} v|^{2}\right. & \left.+s^{2} \varphi_{0}{ }^{2}|v|^{2}\right) e^{2 s \alpha_{0}} \mathrm{~d} x \mathrm{~d} t \\
\leq & C\left(\int_{Q_{\omega}} s^{3} \varphi_{1}^{3}|v|^{2} e^{2 s \alpha_{1}} \mathrm{~d} x \mathrm{~d} t+\left\|\mathrm{e}^{s \alpha_{1}} f\right\|_{\mathrm{L}^{2}(Q)}^{2}+\left\|(\nabla d) s^{-1 / 2} \varphi_{1}^{-1 / 2} e^{s \alpha_{1}}\right\|_{\mathrm{L}^{2}(Q)}^{2}\right) \\
& +C e^{2 s \alpha_{0}}\left(\|v\|_{\mathrm{L}^{2}\left(0, \mathrm{~T} ; \mathrm{H}^{2}(\Omega)\right.}^{2}+\|p\|_{\mathrm{L}^{2}\left(0, \mathrm{~T} ; \mathrm{H}^{1}(\Omega)\right.}^{2}\right) .
\end{aligned}
$$

Then, by dividing inequality (34) by $e^{2 s \alpha_{0}}$ and using (33), we obtain

$$
\begin{aligned}
\int_{Q}\left(s \varphi_{0}|\operatorname{curl} v|^{2}\right. & \left.+s^{2} \varphi_{0}{ }^{2}|v|^{2}\right) \mathrm{d} x \mathrm{~d} t \\
& \leq C\left(\int_{Q_{\omega}} s^{3} \varphi_{1}^{3}|v|^{2} e^{2 s\left(\alpha_{1}-\alpha_{0}\right)} \mathrm{d} x \mathrm{~d} t+e^{2 s\left(\alpha_{1}-\alpha_{0}\right)}\|f\|_{\mathrm{L}^{2}(Q)}^{2}+e^{2 s\left(\alpha_{1}-\alpha_{0}\right)}\left\|(\nabla d) s^{-1 / 2} \varphi_{1}^{-1 / 2}\right\|_{\mathrm{L}^{2}(Q)}^{2}\right) \\
& +C\left(\|v\|_{L^{2}\left(0, \mathrm{~T} ; \mathrm{H}^{2}(\Omega)\right.}^{2}+\|p\|_{\mathrm{L}^{2}\left(0, \mathrm{~T} ; \mathrm{H}^{1}(\Omega)\right.}^{2}\right) .
\end{aligned}
$$

Thus, we have

$$
\begin{aligned}
\int_{Q}|v|^{2} \mathrm{~d} x \mathrm{~d} t & \leq C\left(\int_{Q_{\omega}} s \frac{\varphi_{1}^{3}}{\varphi_{0}^{2}}|v|^{2} e^{2 s\left(\alpha_{1}-\alpha_{0}\right)} \mathrm{d} x \mathrm{~d} t+\frac{e^{2 s\left(\alpha_{1}-\alpha_{0}\right)}}{s^{2} \varphi_{0}^{2}}\|f\|_{\mathrm{L}^{2}(Q)}^{2}+\frac{e^{2 s\left(-\alpha_{1}-\alpha_{0}\right)} \varphi_{1}^{-1}}{s \varphi_{0}^{2}}\|(\nabla d)\|_{\mathrm{L}^{2}(Q)}^{2}\right) \\
& +\frac{C}{s^{2} \varphi_{0}^{2}}\left(\|v\|_{\mathrm{L}^{2}\left(0, \mathrm{~T} ; \mathrm{H}^{2}(\Omega)\right.}^{2}+\|p\|_{\mathrm{L}^{2}\left(0, \mathrm{~T} ; \mathrm{H}^{1}(\Omega)\right.}^{2}\right) \\
& \leq e^{2 s e^{c^{*} \lambda}}\left(\|f\|_{\mathrm{L}^{2}(Q)}^{2}+\|d\|_{\mathrm{L}^{2}\left(0, T ; \mathrm{H}^{1}(\Omega)\right)}^{2}+\|v\|_{\mathrm{L}^{2}\left(Q_{\omega}\right)}^{2}\right)+\frac{1}{s^{2}}\left(\|v\|_{\mathrm{L}^{2}\left(0, \mathrm{~T} ; \mathrm{H}^{2}(\Omega)\right.}^{2}+\|p\|_{\mathrm{L}^{2}\left(0, \mathrm{~T} ; \mathrm{H}^{1}(\Omega)\right.}^{2}\right),
\end{aligned}
$$

with large enough $c^{*}$ (independent of $\lambda$ ). $\sqcup$

From the above theorem, we can show a logarithmic estimate for the solutions of the Stokes equations that prove the inequality (4) of Theorem 1.1.

Theorem 3.1.2. There exists $c^{*}>0$ such that all $\lambda \geq \hat{\lambda}>1$ results in every solution $(v, p) \in \mathrm{L}^{2}\left(0, T ; \mathrm{H}^{2}(\Omega)\right) \cap \mathrm{H}^{1}\left(0, T ; \mathrm{L}^{2}(\Omega)\right) \times \mathrm{L}^{2}\left(0, T ; \mathrm{H}^{1}(\Omega)\right)$

of Eq. (25) such that $\|v\|_{L^{2}\left(0, \mathrm{~T} ; \mathrm{H}^{2}(\Omega)\right)}+\|p\|_{L^{2}\left(0, \mathrm{~T} ; \mathrm{H}^{1}(\Omega)\right)} \leq M$ for some $M>0$ satisfying:

$$
\|v\|_{\mathrm{L}^{2}(\mathrm{Q})} \leq \frac{e^{e^{e^{* \lambda} \lambda} M}}{\ln \left(1+\frac{M}{\|f\|_{\mathrm{L}^{2}(Q)}+\|d\|_{\mathrm{L}^{2}\left(0, T ; \mathrm{H}^{1}(\Omega)\right)}+\|v\|_{\mathrm{L}^{2}\left(Q_{\omega}\right)}}\right)} .
$$


Proof: We introduce $A=\|f\|_{\mathrm{L}^{2}(Q)}+\|d\|_{\mathrm{L}^{2}\left(0, T ; \mathrm{H}^{1}(\Omega)\right)}+\|v\|_{\mathrm{L}^{2}\left(Q_{\omega}\right)}$ and apply Theorem 3.1 for $s>\hat{s}$, and then we can write (32) in the form: $\|v\|_{L^{2}(Q)} \leq e^{s C^{*}} A+\frac{c^{*}}{S} M$, where $C^{*} \stackrel{d e f}{=} e^{c^{*} \lambda}$.

First, if the case $A=0$, since the previous inequality is true for all $s>\hat{s}$, we obtain $\|v\|_{L^{2}(Q)}^{2}=0$ and then (35) holds.

In the following, we assume $A \neq 0$.

We suppose that $\frac{1}{2 C^{*}} \ln \left(1+\frac{M}{A}\right) \geq \hat{s}$ and choose $s=\frac{1}{2 C^{*}} \ln \left(1+\frac{M}{A}\right)$. This yields

$$
\|v\|_{L^{2}(Q)} \leq M\left(\left(1+\frac{M}{A}\right)^{1 / 2} \frac{A}{M}+\frac{2 C^{*} c^{*}}{\ln \left(1+\frac{M}{A}\right)}\right) .
$$

Next, using the fact that

$$
\frac{1}{x} \leq \frac{1}{\ln (1+x)} \text { for } 0<x \leq 1 \text {, i.e } \quad M \leq A \text {, and } \frac{1}{x^{1 / 2}} \leq \frac{1}{\ln (1+x)} \text { for } x>1 \text {, i.e } M>A,
$$

we obtain (35) (by choosing large enough values of $c^{*}>0$ ).

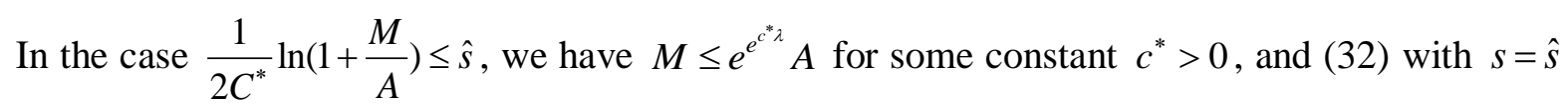
gives $\|v\|_{L^{2}(\mathrm{Q})} \leq e^{e^{e^{*} \lambda}} A$, for some constant $c^{*}>0$. Then the conclusion follows from

$$
A=M \frac{A}{M} \leq M \frac{1}{\ln \left(1+\frac{M}{A}\right)}\left(\text { since } \frac{1}{x} \leq \frac{1}{\ln (1+x)}, \forall x>0\right) .
$$

\section{Estimates for the solutions with a boundary observation}

Theorem 3.2.1. There exists $c^{*}>0$ such that all $\lambda \geq \hat{\lambda}>1$ results in every solution $(v, p) \in \mathrm{L}^{2}\left(0, T ; \mathrm{H}^{2}(\Omega)\right) \cap \mathrm{H}^{1}\left(0, T ; \mathrm{L}^{2}(\Omega)\right) \times \mathrm{L}^{2}\left(0, T ; \mathrm{H}^{1}(\Omega)\right)$ of the Stokes equations (1) associated with an additional observation given by (3) such that $\left(\|v\|_{\mathrm{L}^{2}\left(0, \mathrm{~T} ; \mathrm{H}^{2}(\Omega)\right.}+\|p\|_{\mathrm{L}^{2}\left(0, \mathrm{~T} ; \mathrm{H}^{1}(\Omega)\right.}\right) \leq M$ for some $M>0$ satisfies

$$
\begin{aligned}
\|v\|_{\mathrm{L}^{2}(\mathrm{Q})} & \leq e^{s e^{e^{*} \lambda}}\left(\|f\|_{\mathrm{L}^{2}(Q)}+\|d\|_{\mathrm{L}^{2}\left(0, T ; \mathrm{H}^{1}(\Omega)\right)}+\|v\|_{\mathrm{L}^{2}\left(0, \mathrm{~T} ; \mathrm{H}^{3 / 2}\left(\Gamma_{o b s}\right)\right)}+\|\sigma(v, p) n\|_{\mathrm{L}^{2}\left(0, \mathrm{~T} ; \mathrm{H}^{1 / 2}\left(\Gamma_{o b s}\right)\right)}\right) \\
& +\frac{1}{S}\left(\|v\|_{\mathrm{L}^{2}\left(0, \mathrm{~T} ; \mathrm{H}^{2}(\Omega)\right.}+\|p\|_{\mathrm{L}^{2}\left(0, \mathrm{~T} ; \mathrm{H}^{1}(\Omega)\right.}\right),
\end{aligned}
$$

and

$$
\begin{aligned}
& \|\operatorname{curl}(v)\|_{\left(\mathrm{L}^{2}(Q)\right)^{2 N-3}}+\|p-\operatorname{div}(v)\|_{\mathrm{L}^{2}(Q)} \\
& \quad \leq e^{s e^{e^{*} \lambda}}\left(\|f\|_{\mathrm{L}^{2}(Q)}+\|d\|_{\mathrm{L}^{2}\left(0, T ; \mathrm{H}^{1}(\Omega)\right)}+\|v\|_{\mathrm{L}^{2}\left(0, \mathrm{~T} ; \mathrm{H}^{3 / 2}\left(\Gamma_{o b s}\right)\right)}+\|\sigma(v, p) n\|_{\mathrm{L}^{2}\left(0, \mathrm{~T} ; \mathrm{H}^{1 / 2}\left(\Gamma_{o b s}\right)\right)}\right) \\
& \quad+\frac{1}{s^{1 / 2}}\left(\|v\|_{\mathrm{L}^{2}\left(0, \mathrm{~T} ; \mathrm{H}^{2}(\Omega)\right.}+\|p\|_{\mathrm{L}^{2}\left(0, \mathrm{~T} ; \mathrm{H}^{1}(\Omega)\right.}\right) .
\end{aligned}
$$


Let us begin by proving the following lemma, which is a construction of an extension of the domain $Q$ and of the solution $(v, p)$ of Problem (25). It is deduced from a result of Badra et al. (2016).

Lemma 3.2.2. Let $\tilde{\Omega}$ be an extension of $\Omega$ of class $C^{2}$ through $\Gamma_{\text {obs }}$, namely $\tilde{\Omega}$ is class $C^{2}$, $\partial \Omega \cap \tilde{\Omega}=\Gamma_{o b s}$. We also denote $\tilde{Q}=(0, T) \times \tilde{\Omega}$.

There exists an extension $(\tilde{v}, \tilde{p}) \in \mathrm{L}^{2}\left(0, T ; \mathrm{H}^{2}(\tilde{\Omega})\right) \cap \mathrm{H}^{1}\left(0, T ; \mathrm{L}^{2}(\tilde{\Omega})\right) \times \mathrm{L}^{2}\left(0, T ; \mathrm{H}^{1}(\tilde{\Omega})\right) \quad$ of $(v, p) \in \mathrm{L}^{2}\left(0, T ; \mathrm{H}^{2}(\Omega)\right) \cap \mathrm{H}^{1}\left(0, T ; \mathrm{L}^{2}(\Omega)\right) \times \mathrm{L}^{2}\left(0, T ; \mathrm{H}^{1}(\Omega)\right)$ such that

$$
\left.\tilde{v}\right|_{\Gamma_{o b s}}=\left.v\right|_{\Gamma_{o b s}},\left.\frac{\partial \tilde{v}}{\partial n}\right|_{\Gamma_{o b s}}=\left.\frac{\partial v}{\partial n}\right|_{\Gamma_{o b s}},\left.\tilde{p}\right|_{\Gamma_{o b s}}=\left.p\right|_{\Gamma_{o b s}},
$$

with the following estimate

$$
\begin{aligned}
\|\tilde{v}\|_{H^{1}\left(0, \mathrm{~T} ; \mathrm{H}^{2}(\Omega(\Omega))\right.}^{2}+\|\tilde{p}\|_{\mathrm{L}^{2}\left(0, \mathrm{~T} ; \mathrm{H}^{1}(\Omega \backslash \Omega)\right)}^{2} & \\
\leq C & \left.C\|v\|_{\mathrm{L}^{2}\left(0, \mathrm{~T} ; \mathrm{H}^{3 / 2}\left(\Gamma_{o b s}\right)\right)}^{2}+\left\|\frac{\partial v}{\partial n}\right\|_{L^{2}\left(0, \mathrm{~T} ; \mathrm{H}^{1 / 2}\left(\Gamma_{o b s}\right)\right)}^{2}+\|p\|_{\mathrm{L}^{2}\left(0, \mathrm{~T} ; \mathrm{H}^{1 / 2}\left(\Gamma_{o b s}\right)\right)}^{2}\right) .
\end{aligned}
$$

In particular,

$$
\left(\|v\|_{\mathrm{H}^{1}\left(0, \mathrm{~T} ; \mathrm{H}^{2}(\tilde{\Omega})\right.}^{2}+\|p\|_{\mathrm{L}^{2}\left(0, \mathrm{~T} ; \mathrm{H}^{1}(\tilde{\Omega})\right.}^{2}\right) \leq C\left(\|v\|_{\mathrm{L}^{2}\left(0, \mathrm{~T} ; \mathrm{H}^{2}(\Omega)\right.}^{2}+\|p\|_{\mathrm{L}^{2}\left(0, \mathrm{~T} ; \mathrm{H}^{1}(\Omega)\right.}^{2}\right) .
$$

Proof of Theorem 3.2.1. Let $(\tilde{v}, \tilde{p})$ be an extension of $(v, p)$ as in Lemma 3.2.2.

Let us consider $\omega \subset \tilde{\Omega} \backslash \Omega$ a non-empty bounded open subset, and denote $Q_{\omega}=(0, T) \times \omega$.

Applying (26) and (27) to $(v, p)$ we get

$$
\begin{aligned}
& \int_{\tilde{Q}}\left(s \varphi|\operatorname{curl} \tilde{v}|^{2}+s^{2} \varphi^{2}|\tilde{v}|^{2}+s \varphi|\tilde{p}-\operatorname{div} \tilde{v}|^{2}\right) e^{2 s \alpha} \mathrm{d} x \mathrm{~d} t \\
& \leq C\left(\int_{Q_{\omega}}\left(s^{3} \varphi^{3}|\tilde{v}|^{2}+s \varphi|\tilde{p}-\operatorname{div} \tilde{v}|^{2}\right) e^{2 s \alpha} \mathrm{d} x \mathrm{~d} t+\left\|\mathrm{e}^{s \alpha}\left(\tilde{v}_{t}^{\prime}-\Delta \tilde{v}+\nabla p\right)\right\|_{L^{2}(\tilde{Q})}^{2}+\left\|(\nabla \operatorname{div} \tilde{v}) s^{-1 / 2} \varphi^{-1 / 2} e^{s \alpha}\right\|_{\mathrm{L}^{2}(\tilde{Q})}^{2}\right) \\
& +C e^{2 s \alpha_{0}}\left(\|\tilde{v}\|_{L^{2}\left(0, \mathrm{~T} ; \mathrm{H}^{2}(\Omega)\right.}^{2}+\|\tilde{p}\|_{\mathrm{L}^{2}\left(0, \mathrm{~T} ; \mathrm{H}^{1}(\Omega)\right.}^{2}\right),
\end{aligned}
$$

and then from the estimates (38) and (39) of Lemma 3.2.2 and $\omega \subset \tilde{\Omega} \backslash \Omega$ we deduce

$$
\begin{aligned}
& \int_{Q}\left(s \varphi|\operatorname{curl} v|^{2}+s^{2} \varphi^{2}|v|^{2}+s \varphi|p-\operatorname{div} v|^{2}\right) e^{2 s \alpha} \mathrm{d} x \mathrm{~d} t \\
& \leq C\left(\int_{Q_{\omega}}\left(s^{3} \varphi^{3}|\tilde{v}|^{2}+s \varphi|\tilde{p}-\operatorname{div} \tilde{v}|^{2}\right) e^{2 s \alpha_{1}} \mathrm{~d} x \mathrm{~d} t+\left\|\mathrm{e}^{s \alpha}\left(\tilde{v}_{t}^{\prime}-\Delta \tilde{v}+\nabla \tilde{p}\right)\right\|_{L^{2}(\tilde{Q})}^{2}+\left\|(\nabla \operatorname{div} \tilde{v}) s^{-1 / 2} \varphi^{-1 / 2} e^{s \alpha_{1}}\right\|_{\mathrm{L}^{2}(\tilde{Q})}^{2}\right) \\
& +C e^{2 s \alpha_{0}}\left(\|v\|_{L^{2}\left(0, \mathrm{~T} ; \mathrm{H}^{2}(\Omega)\right.}^{2}+\|p\|_{\mathrm{L}^{2}\left(0, \mathrm{~T} ; \mathrm{H}^{1}(\Omega)\right.}^{2}\right) \\
& \leq C\left(\left\|\mathrm{e}^{s \alpha_{1}} f\right\|_{L^{2}(Q)}^{2}+\left\|(\nabla \operatorname{div} v) e^{s \alpha_{1}}\right\|_{\mathrm{L}^{2}(Q)}^{2}\right) \\
& +C \varphi_{1}^{3} e^{2 s \alpha_{1}} \int_{\tilde{Q} \backslash Q}\left(s^{3}|\tilde{v}|^{2}+\left|\tilde{v}_{t}^{\prime}\right|^{2}+|\Delta \tilde{v}|^{2}+|\nabla \tilde{p}|^{2}+|\nabla \operatorname{div} \tilde{v}|^{2}+s|\tilde{p}-\operatorname{div} \tilde{v}|^{2}\right) d x d t
\end{aligned}
$$




$$
\begin{aligned}
& +C e^{2 s \alpha_{0}}\left(\|v\|_{\mathrm{L}^{2}\left(0, \mathrm{~T} ; \mathrm{H}^{2}(\Omega)\right.}^{2}+\|p\|_{\mathrm{L}^{2}\left(0, \mathrm{~T} ; \mathrm{H}^{1}(\Omega)\right.}^{2}\right) \\
& \quad \leq C\left(\left\|\mathrm{e}^{s \alpha_{1}} f\right\|_{\mathrm{L}^{2}(Q)}^{2}+\left\|(\nabla \operatorname{div}(v)) e^{s \alpha_{1}}\right\|_{\mathrm{L}^{2}(Q)}^{2}\right) \\
& +C s^{3} \varphi_{1}^{3} e^{2 s \alpha_{1}}\left(\|v\|_{\mathrm{L}^{2}\left(0, \mathrm{~T} ; \mathrm{H}^{3 / 2}\left(\Gamma_{o b s}\right)\right)}^{2}+\left\|\frac{\partial v}{\partial n}\right\|_{\mathrm{L}^{2}\left(0, \mathrm{~T} ; \mathrm{H}^{1 / 2}\left(\Gamma_{o b s}\right)\right)}^{2}+\|p\|_{\mathrm{L}^{2}\left(0, \mathrm{~T} ; \mathrm{H}^{1 / 2}\left(\Gamma_{o b s}\right)\right)}^{2}\right) \\
& +C e^{2 s \alpha_{0}}\left(\|v\|_{\mathrm{L}^{2}\left(0, \mathrm{~T} ; \mathrm{H}^{2}(\Omega)\right.}^{2}+\|p\|_{\mathrm{L}^{2}\left(0, \mathrm{~T} ; \mathrm{H}^{1}(\Omega)\right.}^{2}\right),
\end{aligned}
$$

with $\alpha_{1}$ being defined by (33).

Thus, by dividing the inequality (40) by $e^{2 s \alpha_{0}}$ and using that $e^{-2 s \alpha_{0}} \int_{Q} \varphi^{2}|v|^{2} e^{2 s \alpha} \mathrm{d} x \mathrm{~d} t \geq \int_{Q}|v|^{2} \mathrm{~d} x \mathrm{~d} t$, we have

$$
\begin{aligned}
& \|v\|_{\mathrm{L}^{2}(\mathrm{Q})}^{2} \leq \\
& C \frac{\mathrm{e}^{2 s\left(\alpha_{1}-\alpha_{0}\right)} s \varphi_{1}^{3}}{\varphi^{2}}\left(\|f\|_{\mathrm{L}^{2}(Q)}^{2}+\|(\nabla \operatorname{div} v)\|_{\mathrm{L}^{2}(Q)}^{2}+\|v\|_{\mathrm{L}^{2}\left(0, \mathrm{~T} ; \mathrm{H}^{3 / 2}\left(\Gamma_{o b s}\right)\right)}^{2}+\left\|\frac{\partial v}{\partial n}\right\|_{\mathrm{L}^{2}\left(0, \mathrm{~T} ; \mathrm{H}^{1 / 2}\left(\Gamma_{o b s}\right)\right)}^{2}+\|p\|_{\mathrm{L}^{2}\left(0, \mathrm{~T} ; \mathrm{H}^{1 / 2}\left(\Gamma_{o b s}\right)\right)}^{2}\right) \\
& +\frac{C}{s^{2} \varphi^{2}}\left(\|v\|_{\mathrm{L}^{2}\left(0, \mathrm{~T} ; \mathrm{H}^{2}(\Omega)\right.}^{2}+\|p\|_{\mathrm{L}^{2}\left(0, \mathrm{~T} ; \mathrm{H}^{1}(\Omega)\right.}^{2}\right) .
\end{aligned}
$$

We note that from the definition of $\sigma(v, p) n$, it is possible to replace the term $\left\|\frac{\partial v}{\partial n}\right\|_{L^{2}\left(0, \mathrm{~T} ; \mathrm{H}^{1 / 2}\left(\Gamma_{o b s}\right)\right)}^{2}+\|p\|_{L^{2}\left(0, \mathrm{~T} ; \mathrm{H}^{1 / 2}\left(\Gamma_{o b s}\right)\right)}^{2}$ in the last inequality with $\|\sigma(v, p) n\|_{\mathrm{L}^{2}\left(0, \mathrm{~T} ; \mathrm{H}^{1 / 2}\left(\Gamma_{o b s}\right)\right)}^{2}$, so we get

$$
\begin{aligned}
& \|v\|_{\mathrm{L}^{2}(\mathrm{Q})}^{2} \leq e^{2 s e^{e^{* \lambda} \lambda}}\left(\|f\|_{\mathrm{L}^{2}(Q)}^{2}+\|d\|_{\mathrm{L}^{2}\left(0, T ; \mathrm{H}^{1}(\Omega)\right)}^{2}+\|v\|_{\mathrm{L}^{2}\left(0, \mathrm{~T} ; \mathrm{H}^{3 / 2}\left(\Gamma_{o b s}\right)\right)}^{2}+\|\sigma(v, p) n\|_{\mathrm{L}^{2}\left(0, \mathrm{~T} ; \mathrm{H}^{1 / 2}\left(\Gamma_{o b s}\right)\right)}^{2}\right) \\
& +\frac{1}{s^{2}}\left(\|v\|_{\mathrm{L}^{2}\left(0, \mathrm{~T} ; \mathrm{H}^{2}(\Omega)\right.}^{2}+\|p\|_{\mathrm{L}^{2}\left(0, \mathrm{~T} ; \mathrm{H}^{1}(\Omega)\right.}^{2}\right) .
\end{aligned}
$$

With a similar argument as above, we can prove that

$$
\begin{aligned}
& \|\operatorname{curl}(v)\|_{\left.\mathrm{L}^{2}(Q)\right)^{2 N-3}}^{2}+\|p-\operatorname{div}(v)\|_{\mathrm{L}^{2}(Q)}^{2} \\
& \leq e^{2 s e^{e^{*} \lambda}}\left(\|f\|_{\mathrm{L}^{2}(Q)}^{2}+\|d\|_{\mathrm{L}^{2}\left(0, T ; \mathrm{H}^{1}(\Omega)\right)}^{2}+\|v\|_{\mathrm{L}^{2}\left(0, \mathrm{~T} ; \mathrm{H}^{3 / 2}\left(\Gamma_{o b s}\right)\right)}^{2}+\|\sigma(v, p) n\|_{\mathrm{L}^{2}\left(0, \mathrm{~T} ; \mathrm{H}^{1 / 2}\left(\Gamma_{o b s}\right)\right)}^{2}\right) \\
& +\frac{1}{S}\left(\|v\|_{\mathrm{L}^{2}\left(0, \mathrm{~T} ; \mathrm{H}^{2}(\Omega)\right.}^{2}+\|p\|_{\mathrm{L}^{2}\left(0, \mathrm{~T} ; \mathrm{H}^{1}(\Omega)\right.}^{2}\right) .
\end{aligned}
$$

The estimates (41) and (42) directly lead to (36) and (37).

With the help of Theorem 3.2.1 and using similar arguments of the proof of Theorem 3.1.2, we have the following results, which prove the inequalities (5) and (6) of Theorem 1.1.

Theo rem 3.2.3. Assume that $(v, p) \in L^{2}\left(0, \mathrm{~T} ; \mathrm{H}^{2}(\Omega)\right) \cap H^{1}\left(0, \mathrm{~T} ; L^{2}(\Omega)\right) \times L^{2}\left(0, \mathrm{~T} ; \mathrm{H}^{1}(\Omega)\right)$ is the solution of the Stokes equation (1) associated with an additional observation given by (3) such that $\left(\|v\|_{\mathrm{L}^{2}\left(0, \mathrm{~T} ; \mathrm{H}^{2}(\Omega)\right.}+\|p\|_{\mathrm{L}^{2}\left(0, \mathrm{~T} ; \mathrm{H}^{1}(\Omega)\right.}\right) \leq M$ for some $M>0$. Then there exists a constant $C>0$ such that we have the following estimates: 


$$
\|v\|_{\mathrm{L}^{2}(Q)} \leq C \frac{M}{\ln \left(1+\frac{M}{\|f\|_{\mathrm{L}^{2}(Q)}+\|d\|_{\mathrm{L}^{2}(Q)}+\|v\|_{\mathrm{L}^{2}\left(0, T ; H^{3 / 2}\left(\Gamma_{o b s}\right)\right)}+\|\sigma(v, p) \mathrm{n}\|_{\mathrm{L}^{2}\left(0, T ; H^{1 / 2}\left(\Gamma_{o b s}\right)\right)}}\right)} .
$$

Moreover, we have

$$
\begin{aligned}
& \|\operatorname{curl}(v)\|_{\left(\mathrm{L}^{2}(Q)\right)^{2 N-3}}+\|p-\operatorname{div}(v)\|_{\mathrm{L}^{2}(Q)} \\
& \leq C \frac{M}{\left[\ln \left(1+\frac{M}{\|f\|_{L^{2}(Q)}+\|d\|_{L^{2}(Q)}+\|v\|_{L^{2}\left(0, T ; H^{3 / 2}\left(\Gamma_{o b s}\right)\right)}+\|\sigma(v, p) \mathrm{n}\|_{L^{2}\left(0, T ; H^{1 / 2}\left(\Gamma_{o b s}\right)\right)}}\right)\right]^{\frac{1}{2}}}
\end{aligned}
$$

\section{Stability of solutions: the proof of Theorem 1.2}

This section focuses on the proof of Theorem 1.2, which shows the stability of the solutions of the Stokes equations.

Let $(v, p)=\left(v_{1}-v_{2}, p_{1}-p_{2}\right)$, then it is the solution to

$$
\left\{\begin{array}{rlrlrl}
v_{t}^{\prime}-\Delta v+\nabla p & =f & & \text { in } & & Q \\
\operatorname{div}(v) & =0 & & \text { in } & & Q \\
v(0, x) & =v_{0}(x) & \text { for } & & x \in \Omega
\end{array} .\right.
$$

This equation is a particular form of Eq. (1). The additional observation for the pair $(v, p)$ is:

$$
\begin{aligned}
& v=v_{1}-v_{2} \text { in }(0, \mathrm{~T}) \times \omega,(44) \text { or } \\
& \left\{\begin{array}{cl}
v=v_{1}-v_{2} & \text { on }(0, \mathrm{~T}) \times \Gamma_{o b s} . \\
\sigma(v, p) \mathrm{n}=\sigma\left(v_{1}, p_{1}\right) \mathrm{n}-\sigma\left(v_{2}, p_{2}\right) \mathrm{n} & \text { on } \quad(0, \mathrm{~T}) \times \Gamma_{o b s}
\end{array}\right.
\end{aligned}
$$

By applying Theorem 1.1 for the pair $(v, p)$, we directly get the estimates (9), (10), and (11).

\section{Stability of the Robin coefficients: the proof of Theorem 1.3}

In this section, we show the stability of the Robin coefficients in the Stokes equations with the help of the stability of the solutions. We focus on the proof of Theorem 1.3.

Let $\left(v_{i}, p_{i}\right), i=1,2$, be two solutions of Eq. (1) associated with the additional observation given by (8) and let $\alpha_{i}, i=1,2$ be the two Robin coefficients given by (12).

As in the proof of Theorem 1.2 shown above, let $(v, p)=\left(v_{1}-v_{2}, p_{1}-p_{2}\right)$, which is the solution to Eq. (43) with the additional observation given by (45).

Without a loss of generality, we can assume that $\left|v_{1}\right| \geq m$. The Robin coefficients given by (8) then satisfy the relationship:

$$
\left(\alpha_{1}-\alpha_{2}\right) v_{1}=\alpha_{2} v+\sigma(v, p) \mathrm{n}=\alpha_{2} v+(\eta D(v)-p I) n \text { on } \Gamma_{0} .
$$

Thus, we get the estimate

$$
\left\|\alpha_{1}-\alpha_{2}\right\|_{L^{2}((0, T) \times \mathrm{K})} \leq \frac{C}{m}\left(\|v\|_{\mathrm{L}^{2}\left((0, T) \times \Gamma_{0}\right)}+\|\nabla v\|_{\mathrm{L}^{2}\left((0, T) \times \Gamma_{0}\right)}+\|p\|_{\mathrm{L}^{2}\left((0, T) \times \Gamma_{0}\right)}\right) .
$$

Using an interpolation inequality borrowed from Badra et al. (2016) $\|\cdot\|_{L^{2}(\partial \Omega)} \leq C\|\cdot\|_{L^{2}(\Omega)}\|\cdot\|_{H^{1}(\Omega)}$, we can get 


$$
\begin{aligned}
& \|p\|_{L^{2}\left((0, T) \times \Gamma_{0}\right)} \leq C\|p\|_{L^{2}(Q)}^{1 / 2}\|p\|_{L^{2}\left(0, \mathrm{~T} ; \mathrm{H}^{1}(\Omega)\right)}^{1 / 1}, \\
& \|v\|_{L^{2}\left((0, T) \times \Gamma_{0}\right)} \leq C\|v\|_{L^{2}(Q)}^{1 / 2}\|v\|_{L^{2}\left(0, \mathrm{~T} ; \mathrm{H}^{1}(\Omega)\right)}^{1 / 2}, \\
& \text { and then }\|\nabla v\|_{L^{2}\left((0, T) \times \Gamma_{0}\right)} \leq C\|v\|_{L^{2}\left(0, \mathrm{~T} ; \mathrm{H}^{1}(\Omega)\right)}^{1 / 2}\|v\|_{L^{2}\left(0, \mathrm{~T} ; \mathrm{H}^{2}(\Omega)\right)}^{1 / 2} .
\end{aligned}
$$

Combining (47), (48), and (49) with the interpolation inequality $\|\cdot\|_{H^{1}(\Omega)} \leq\|\cdot\|_{L^{2}(\Omega)}^{1 / 2}\|\cdot\|_{H^{2}(\Omega)}^{1 / 2}$, the inequality (46) then leads to

$$
\begin{aligned}
\left\|\alpha_{1}-\alpha_{2}\right\|_{\mathrm{L}^{2}((0, T) \times \mathrm{K})} & \leq \frac{C}{m}\left(\|v\|_{\mathrm{L}^{2}(Q)}^{3 / 4}\|v\|_{L^{2}\left(0, \mathrm{~T} ; \mathrm{H}^{2}(\Omega)\right)}^{1 / 4}+\|v\|_{\mathrm{L}^{2}(Q)}^{1 / 4}\|v\|_{L^{2}\left(0, \mathrm{~T} ; \mathrm{H}^{2}(\Omega)\right)}^{3 / 4}+\|p\|_{\mathrm{L}^{2}(Q)}^{1 / 2}\|p\|_{L^{2}\left(0, \mathrm{~T} ; \mathrm{H}^{1}(\Omega)\right)}^{1 / 2}\right) \\
& \leq \frac{C}{m}\left(\|v\|_{\mathrm{L}^{2}(Q)}^{1 / 4}\|v\|_{L^{2}\left(0, \mathrm{~T} ; \mathrm{H}^{2}(\Omega)\right)}^{3 / 4}+\|p\|_{\mathrm{L}^{2}(Q)}^{1 / 2}\|p\|_{L^{2}\left(0, \mathrm{~T} ; \mathrm{H}^{1}(\Omega)\right)}^{1 / 2}\right) \\
& \leq \frac{C}{m}\left(\|v\|_{\mathrm{L}^{2}(Q)}^{1 / 4} M^{3 / 4}+\|p\|_{\mathrm{L}^{2}(Q)}^{1 / 2} M^{1 / 2}\right)
\end{aligned}
$$

Hence, the result (13) of Theorem 1.3 is proven by applying Theorem 1.2 (the inequalities (10) and (11)) to the estimate $\|v\|_{L^{2}(Q)}$ and $\|p\|_{L^{2}(Q)}$ in the above inequality (50).

We notice from the result of Theorem 1.3 that if the boundary observations of $v_{1}$ and $v_{2}$ on the observable boundary part are equal, then the corresponding Robin coefficients $\alpha_{1}$ and $\alpha_{2}$ are also equal.

\section{Discussion}

The estimates (9), (10), and (11) (stated in Theorem 1.2) show the stability of the velocity and of the pair of solutions $(v, p)$ with respect to a distributed observation or a boundary observation. These results extend the results of Boulakia et al. (2013) and Badra et al. (2016) by building an appropriate Carleman estimate for the unsteady Stokes Eq. (1).

On the other hand, the uniqueness of the solution $(v, p)$ of Eq. (1) is guaranteed thanks to the above results: if $v_{1}=v_{2}$ in $Q_{\omega}$ then $v_{1}=v_{2}$ in $Q$, or if the Cauchy data $\left(v_{1}, \sigma\left(v_{1}, p_{1}\right) \mathrm{n}\right)=$ $\left(v_{2}, \sigma\left(v_{2}, p_{2}\right) \mathrm{n}\right)$ on $(0, \mathrm{~T}) \times \Gamma_{\text {obs }}$, then $v_{1}=v_{2}$ in $Q$. This matches the unique continuation result given in Fabre and Lebeau (1996).

The estimate (13) shows the stability in the determination of the Robin boundary coefficient from the value of the velocity $v$ and the Cauchy data $(v, \sigma(v, p) n)$ on a part of the boundary. This means that the Robin coefficient can be determined from an unobservable boundary part of the velocity and of the Cauchy data.

Many mathematical works dealing with boundary coefficient problems have previously been published (Chaabane et al., 2004; Sincich, 2007; Bellassoued et al., 2008; Cheng et al., 2008; Boulakia et al., 2013). However, the present work is for unsteady Stokes equations in two or three dimensions.

\section{Conclusions}

The present work treats, for the first time, the inverse problem of determining the timeindependent Robin coefficient in unsteady Stokes equations (in 2 or 3 dimensions) with non-homogeneous boundary conditions.

The stability estimates of a log type are established for the solution of the equations from either an interior measurement of the velocity, or a boundary observation depending on the trace of the velocity and of the Cauchy stress tensor measurements on a part of the boundary. 
A stability estimate for the Robin coefficient is then established from a measurement of the solution and of the Cauchy data on a sub-boundary. It is very significant in the determination of the Robin coefficient on the inaccessible portion of the boundary thanks to electrostatic measurements performed on the accessible boundary part.

\section{Acknowledgements}

We would like to thank the Vietnam National University of Agriculture and the Faculty of Information Technology for their support, which helped us to complete this work.

\section{References}

Adams R. A. and Fournier J. (2003). Sobolev spaces. Pure and Applied Mathematics. New York - London: Acadmic Press. Vol 140.

Badra M., Canbet F. and Dare J. (2016). Stability estimates for Navier - Stokes equations and application to inverse problems. Discrete and Continuous Dynamical Systems - Series B. Vol 21 (8). pp. 2379-2407.

Baffico L., Grandmont C. and Maury B. (2010). Multiscale modeling of the respiratory tract. Mathematical Models and Methods in Applied Sciences. Vol 20 (1). pp. 59-93.

Bellassoued M., Cheng J. and Choulli M. (2008). Stability estimate for an inverse boundary coefficient problem in thermal imaging. Journal of Mathematical Analysis and Applications. Vol 343 (1). pp. 328-336.

Boulakia M., Egloffe A. C. and Grandmont C. (2013). Stability estimates for a Robin coefficient in the Twodimensional Stokes system. Mathematical Control \&
Related Fields. Vol 3 (1). pp. 21-49.

Bramble L. H. (2003). A proof of the inf-sup condition for the Stokes equations on Lipschitz domains. Mathematical Models and Methods in Applied Sciences. Vol 13 (3). pp. 361-371.

Chaabane S., Fellah I., Jaoua M. and Leblond J. (2004). Logarithmic stability estimates for a Robin coefficient in two-dimensional Laplace inverse problems. Inverse Problems. Vol 20 (1). pp. 47-59.

Cheng J., Choulli M. and Lin J. (2008). Stable determination of a boundary coefficient in an elliptic equation. Mathematical Models and Methods in Applied Sciences. Vol 18 (1). pp. 107-123.

Dusenbery D. B. (2011). Living at Micro Scale. Harvard University Press. Cambridge, MA 02138, USA.

Fabre C. and Lebeau G. (1996). Prolongement unique des solutions. Communications in Partial Differential Equations. Vol 21 (3-4). pp. 573-596.

Imanuvilov O. Y. and Yamamoto M. (2003). Carleman Inequalities for Parabolic Equations in Sobolev Spaces of Negative Order and Exact Controllability for semilinear Parabolic Equations. Publications of the Research Institute for Mathematical Sciences. Vol 39 (2). pp. 227-274.

Tucsnak M. and Weiss G. (2009). Observation and control for operator semigroups. Birkhauser Verlag: Birkhauser Advabced texts.

Necas J. (2012). Direct Methods in the Theory of Elliptic Equations. New york: Springer-Verlag Berlin Heidelberg.

Sincich E. (2007). Lipschitz stability for the inverse Robin problem. Inverse Problems. Vol 23 (3). pp. 1311-1326.

Vignon-Clementel I. E., Figueroa C. A., Jansen K. E. and Taylor C. A. (2006). Outflow boundary conditions for three-dimensional finite element modeling of blood flow and pressure in arteries. Computer Methods in Applied Mechanics and Engineering. Vol 195 (2932). pp. 3776-3796. 